\title{
Hesperetin-loaded lipid-core nanocapsules in polyamide: a new textile formulation for topical drug delivery
}

This article was published in the following Dove Press journal:

International Journal of Nanomedicine

15 March 2017

Number of times this article has been viewed

\author{
Paula dos Passos Menezes' \\ Luiza Abrahão Frank² \\ Bruno dos Santos Lima' \\ Yasmim Maria Barbosa \\ Gomes de Carvalho' \\ Mairim Russo Serafini' \\ Lucindo José \\ Quintans-Júnior ${ }^{3}$ \\ Adriana Raffin Pohlmann ${ }^{4}$ \\ Sílvia Stanisçuaski \\ Guterres $^{2}$ \\ Adriano Antunes de \\ Souza Araújo' \\ 'Department of Pharmacy, Federal \\ University of Sergipe, São Cristóvão, \\ Sergipe, Brazil; ${ }^{2}$ College of Pharmacy, \\ Federal University of Rio Grande do \\ Sul, Porto Alegre, Rio Grande do Sul, \\ Brazil; 'Department of Physiology, \\ Federal University of Sergipe, São \\ Cristóvão, Sergipe, Brazil; ${ }^{4}$ Institute \\ of Chemistry, Federal University of \\ Rio Grande do Sul, Porto Alegre, Rio \\ Grande do Sul, Brazil
}

Correspondence: Paula dos Passos Menezes; Adriano Antunes de Souza Araújo

Department of Pharmacy, Federal University of Sergipe, Marechal Rondon Avenue, Jardim Rosa Elze, São Cristóvão, Sergipe 49100-000, Brazil

Tel +55 7921056841

Fax +557921056827

Email paula.dp.menezes@gmail.com; adriasa200I@yahoo.com.br

\begin{abstract}
Chronic venous insufficiency is characterized by chronic reflux disorder of blood from the peripheral to the central vein, with subsequent venous hypertension and resulting changes in the skin. Traditionally, nonsurgical treatments relied on the use of compression therapy, and more recently a variety of flavonoids have been shown to have positive effects. There have also been developments of more effective drug delivery systems using various textiles and nanotechnology to provide new therapeutic options. Our objective was to use nanotechnology to develop a new formulation containing hesperetin (Hst), a substance not previously used in the treatment of chronic venous insufficiency, impregnated into textile fibers as a possible alternative treatment of venous diseases. We prepared the nanocapsules using the interfacial deposition of preformed polymer method with an Hst concentration of $0.5 \mathrm{mg} / \mathrm{mL}$ and then characterized the size and distribution of particles. To quantify the Hst in the samples, we developed an analytical method using high-performance liquid chromatography. Studies of encapsulation efficiency $(98.81 \% \pm 0.28 \%)$, microscopy, drug release (free-Hst: $104.96 \% \pm 12.83 \%$; lipid-core nanocapsule-Hst: $69.90 \% \pm 1.33 \%$ ), penetration/permeation, drug content $(0.46 \pm 0.01 \mathrm{mg} / \mathrm{mL})$ and the effect of washing the textile after drug impregnation were performed as part of the study. The results showed that nanoparticles of a suitable size and distribution with controlled release of the drug and penetration/permeation into the skin layers were achieved. Furthermore, it was established that polyamide was able to hold more of the drug, with a 2.54 times higher content than the cotton fiber; after one wash and after five washes, this relation was 2.80 times higher. In conclusion, this is a promising therapeutic alternative to be further studied in clinical trials.
\end{abstract}

Keywords: lipid-core nanocapsules, polymer, hesperetin, fabrics, impregnation, medical textiles, polyamide, poly( $\varepsilon$-caprolactone)

\section{Introduction}

Flavonoids are a large group of phenolic compounds that are widely distributed in plants. ${ }^{1}$ Hesperetin (Hst), 5,7,3'-trihydroxy-4'-methoxyflavanone, is a natural bioflavonoid found abundantly in citrus fruits with promising antioxidant and antiinflammatory properties. ${ }^{2,3}$ Several studies have shown that citrus flavonoids can exert antiatherogenic and vasculoprotective effects by modulating the expression of molecules related to inflammation, preventing the formation of foam cells, reducing the area of atherosclerotic plaques, improving the lipid profile, inhibiting the adhesion of monocytes to the endothelium and modulating cell migration. ${ }^{4}$ Furthermore, Hst via the inhibition of L-type voltage-gated $\mathrm{Ca}^{2+}$ channels and the enhancement of voltage-gated $\mathrm{K}^{+}$channel currents of the myocytes has a direct vasorelaxant effect. ${ }^{5}$ In this regard, activation and 
subsequent platelet aggregation are considered very important phenomena in the pathogenesis of cardiovascular and peripheral vascular diseases. It has been demonstrated that collagen- and arachidonic-acid-induced platelet aggregation is potently inhibited by Hst via the inhibition of phospholipase C- $\gamma 2$ (PLC $\gamma 2$ ) phosphorylation and cyclooxygenase- $1{ }^{6}$ Serotonin is also effectively involved in platelet aggregation. Hst can also have antiplatelet effects via the inhibition of arachidonic acid-induced serotonin secretion. ${ }^{1}$ Thus, its antiplatelet effects make Hst a good candidate in the treatment of chronic venous insufficiency (CVI).

However, the clinical use of Hst has been limited due to its low solubility in water and poor in vivo bioavailability. The poor bioavailability of hydrophobic drugs demands the use of high doses to overcome subtherapeutic levels in plasma. ${ }^{7}$ To solve this limitation, a large number of studies have been published describing new delivery systems such as inclusion complexes with cyclodextrins ${ }^{8-11}$ and nanoparticles. ${ }^{7,12-15}$ Nanoparticle systems have been considered promising carriers of therapeutic drugs, and the application of these particles as carriers of natural compounds has been extensively studied over the past few years. ${ }^{16}$

One of the main challenges in relation to chronic diseases is patient adherence to prescribed treatments. Many conditions require care based on a large extent on patient self-management (usually requiring complex multitherapies). Medical technologies that monitor changes in the patient's lifestyle are fundamental to treatment. The World Health Organization has estimated that in developed countries only $50 \%$ of patients who suffer from chronic diseases adhere to prescribed treatments. ${ }^{17}$ From this perspective, nanotechnology is an important tool in the design of new formulations or health care products. ${ }^{11,17}$

Nanoparticles used as drug delivery systems have particle sizes in the range of $10-1,000 \mathrm{~nm} .{ }^{18}$ Depending on the methods and materials used for preparation, different supramolecular structures can be obtained. In general, when composed of an oily core surrounded by a polymer involucre, they are known as nanocapsules. ${ }^{18-21}$ These systems have several advantages, including controlled and sustained release of the drug, allowing a more effective and less toxic treatment than when conventional options are used, and promoting increased efficacy. ${ }^{20}$ On the skin, the nanocapsules can act as a reservoir system for lipophilic drugs, ${ }^{22}$ controlling their penetration/permeation into the skin through contact with the stratum corneum. ${ }^{18}$ Several studies have evaluated the administration of nanocapsules by different routes, namely oral, ocular, cutaneous, vaginal and parenteral, demonstrating the versatility of the systems. ${ }^{12}$ Lipid-core nanocapsules (LNCs) are a very specific kind of nontoxic polymeric nanocapsules, ${ }^{14}$ in which the oily core is formed by an organogel composed of capric/caprylic triglyceride and sorbitan monostearate. The core chemical composition allows these nanocapsules to control drug penetration in different tissues. ${ }^{13}$ A variety of nanosystems such as nanostructured lipid carriers, nanosuspensions and nanocrystals have been studied with Hst, ${ }^{15,23,24}$ but the current study proposes the encapsulation of Hst in LNCs.

New formulations have attracted the textile sector and encouraged the development of fabrics containing drugs or active ingredients for topical delivery in a variety of uses, including the treatment of CVI and other conditions, ${ }^{25,26}$ as well as in antibacterial and insect-repelling textiles. ${ }^{27,28}$ The drug is released in response to a skin stimulus, such as sweating, friction or skin enzymes. ${ }^{29}$ Our aim was to design an innovative approach to the treatment of CVI based on a synergic new fabric containing Hst-loaded LNCs, which would be simple and easy to use and therefore improve adherence to therapy. The efficacy of this promising strategy was evaluated by in vitro studies of release and penetration/ permeation in cutaneous porcine skin.

\section{Materials and methods Materials}

Cotton and polyamide (PA) were obtained from Empório das Sedas (Brazil), polysorbate 80 from Delaware (Porto Alegre, Brazil), poly( $\varepsilon$-caprolactone) (PCL; molecular weight $(\mathrm{Mn})=80,000 \mathrm{~g} / \mathrm{mol})$ and sorbitan monostearate from Aldrich (Lesquin, France), caprylic/capric triglyceride from Brasquim (Brazil), Savon de Marseille from L'Occitane (Brazil) and Hst from Sigma-Aldrich (St Louis, MO, USA).

\section{Analysis by high-performance liquid chromatography-diode array detector (HPLC-DAD)}

For the chromatographic analysis, a standard solution $(100 \mu \mathrm{g} / \mathrm{mL})$ of Hst $\left(\mathrm{C}_{16} \mathrm{H}_{14} \mathrm{O}_{6}\right.$; $\left.\geq 98.0 \%\right)$ in acetonitrile was prepared. The HPLC analyses were performed using an HPLC system that consisted of a degasser DGU-20A3, a SIL-20A HT auto injector, two LC-20AD pumps, an SPDM20A photodiode array detector (DAD), a CTO-20A column oven and a CBM-20A system controller (Shimadzu Co., Kyoto, Japan), equipped with a column of $4.6 \times 150 \mathrm{~mm}^{2}$ ( $5 \mu \mathrm{m}$ particle size). The injection volume was $20 \mu \mathrm{L}$ with a flow rate of $1.0 \mathrm{~mL} / \mathrm{min}$. The mobile phase consisted of 
acetonitrile/water/acetic acid $0.1 \%(50: 49: 1, \mathrm{v} / \mathrm{v} / \mathrm{v})$. The detector was set at $290 \mathrm{~nm}$ for acquiring chromatograms.

\section{Method validation}

The HPLC analysis method was validated with regard to the parameters of linearity, limit of detection (LOD), limit of quantification (LOQ), precision, accuracy and robustness according to the International Conference on Harmonization (ICH) guidance. Linearity was established by evaluating the value of $r$ (correlation coefficient) in the calibration curve of five concentrations $(1.0-20.0 \mu \mathrm{g} / \mathrm{mL})$. LOD and LOQ were determined by the signal-to-noise ratio $(\mathrm{S} / \mathrm{N})$ method, in which an $\mathrm{S} / \mathrm{N}$ ratio of 3 for LOD and an $\mathrm{S} / \mathrm{N}$ ratio of 10 for LOQ were used. The precision of the method was evaluated by performing the intra- and interday variability test. The intraday precision was performed by analyzing nine replicates of Hst $(20 \mu \mathrm{g} / \mathrm{mL})$ on the same day. The interday precision was determined over the course of two consecutive days with six samples per day of Hst $(20 \mu \mathrm{g} / \mathrm{mL})$. Precision was determined as relative standard deviation (RSD\%). Accuracy was performed by analyzing three different Hst samples in three different concentrations: 1 (low), 10 (medium) and 20 (high) $\mu \mathrm{g} / \mathrm{mL}$. Accuracy was evaluated by average percentage accuracy $(\mathrm{A} \%$ ) and $\mathrm{RSD} \%$. The robustness of the method was evaluated by changing different analytical columns $(250 \times 4.6 \mathrm{~mm}$ and $150 \times 4.6 \mathrm{~mm})$ and flow rate $(0.9$, 1.0 and $1.1 \mathrm{~mL} / \mathrm{min}$ ) in triplicate.

\section{Preparation of nanocapsules}

The LNCs were prepared by the interfacial deposition of preformed polymer method. ${ }^{30}$ The polymer, PCL $(0.1 \mathrm{~g})$, sorbitan monostearate $(0.038 \mathrm{~g})$, capric/caprylic triglyceride $(0.165 \mathrm{~mL})$ and Hst $(0.005 \mathrm{~g})$ were dissolved in acetone $(27 \mathrm{~mL})$. In another flask, polysorbate 80 (0.077 g) was dispersed in $53 \mathrm{~mL}$ of water. The organic and the aqueous phases were heated to $40^{\circ} \mathrm{C}$, under stirring. After dissolving all the components, the organic phase was injected into the aqueous phase and the instantaneously formed turbid solution was kept under magnetic stirring at room temperature for $10 \mathrm{~min}$. The final volume of the formulation was adjusted to $10 \mathrm{~mL}$, after eliminating the organic solvent, acetone and part of the water under reduced pressure at $40^{\circ} \mathrm{C}$.

\section{Particle characterization}

Particle size and zeta potential

Laser diffraction analysis was performed using Mastersizer ${ }^{\circledR}$ 2000 (Malvern, UK) to characterize the particle size distribution in the range from $40 \mathrm{~nm}$ to $2 \mathrm{~mm}$. Volume-weighted mean diameter $\left(\mathrm{D}_{4,3}\right)$ was determined using the Mie theory, and the polydispersity (span) was calculated using the values of diameters at 10, 50 and $90 \%$ under the cumulative size distribution curve by volume $\left(d_{0.1}, d_{0.5}\right.$ and $d_{0.9}$, respectively).

Photon correlation spectroscopy was carried out in a Zetasizer ${ }^{\circledR}$ nano-ZS ZEN 3600 model (Malvern) to characterize the particle size distribution from 0.6 to $2,000 \mathrm{~nm}$. Hydrodynamic mean diameter (z-average diameter) and polydispersity index (PDI) were determined using the method of cumulants. The formulations were diluted in water (ultrapure) for analysis. Zeta potential was determined by electrophoretic light scattering using the same instrument. The samples were diluted in $10 \mathrm{mmol} / \mathrm{L} \mathrm{NaCl}$ aqueous solution.

Three replicates of each formulation were measured at room temperature. Results are presented as mean \pm standard deviation.

\section{$\mathrm{pH}$ determination}

For $\mathrm{pH}$ determination, a calibrated potentiometer DM-22 (Digimed, São Paulo, Brazil) was used. The pH values were measured by the direct immersion of the electrode in the undiluted dispersion. The samples were stored in amber glass flasks at room temperature.

\section{Content determination of samples}

Drug content $(\mathrm{mg} / \mathrm{mL})$ was determined after extraction of the drug from the nanocapsules $(200 \mu \mathrm{L})$ using acetonitrile $(10 \mathrm{~mL})$. The samples were maintained in ultrasound for $60 \mathrm{~min}$. Each dispersion was then centrifuged at $17,632 \times g$ for $15 \mathrm{~min}$ before the supernatant was filtrated and analyzed by HPLC. All measurements were made in triplicate batches.

\section{Encapsulation efficiency (EE)}

The EE of nanocapsules $(400 \mu \mathrm{L})$ was determined by an ultrafiltration-centrifugation technique (Ultrafree-MC 10,000 Da; Millipore, Darmstadt, Germany) at 7,280× $g$ for $10 \mathrm{~min}$. The encapsulated Hst was calculated from the difference between the total and the free drug concentrations determined in the nanocapsule suspension and in the ultrafiltrate, respectively. All measurements were made in triplicate, and samples were analyzed by HPLC.

\section{Transmission electron microscopy}

Diluted (5x-Milli- $\left.\mathrm{Q}^{\circledR}\right)$ formulations were deposited $(10 \mu \mathrm{L})$ on specimen grids (Formvar-carbon support film, electron microscopy sciences) and negatively stained with uranyl 
acetate solution $(2 \% \mathrm{w} / \mathrm{v})$. Analyses were performed using a transmission electron microscope (TEM; JEOL 1230) operating at $80 \mathrm{kV}$ (Serviço de Microscopia Eletrônica from the Fundação Oswaldo Cruz; Fiocruz, Salavador, Brazil).

\section{In vitro release study}

The release profiles of the nanocapsules and the free drug (Hst: $0.5 \mathrm{mg} / \mathrm{mL}$ in an aqueous solution of $10 \%$ ethanol $[\mathrm{v} / \mathrm{v}])$ were determined using the dialysis bag method. ${ }^{21}$ In this study, 10 different release media were tested. An aqueous solution of $10 \%$ ethanol (v/v) showed the best performance in terms of both the saturation concentration of Hst and maintenance of sink condition. Dialysis bags (MW cutoff of $14,000 \mathrm{Da}$, cellulose membrane, average flat width of $10 \mathrm{~mm}$; Sigma-Aldrich) containing $1 \mathrm{~mL}$ of the sample were placed into closed glass flasks containing $100 \mathrm{~mL}$ of the release medium (an aqueous solution of ethanol, 10\%). The system was maintained under constant and moderate agitation in a water bath $\left(32^{\circ} \mathrm{C} \pm 0.5^{\circ} \mathrm{C}\right)$. At fixed time intervals up to $24 \mathrm{~h}$ $(2-24 \mathrm{~h}), 1 \mathrm{~mL}$ of aliquots was withdrawn and replaced by fresh medium. The samples were analyzed by HPLC, and the cumulative drug release was determined.

The data obtained were fitted to the mathematical semiempirical monoexponential and biexponential models using Scientist $^{\circledR} 2.0$ software (MicroMath $^{\circledR}$, St Louis, MO, USA). The fits were analyzed considering the correlation between experimental points and theoretical profiles and the higher value of model selection criteria (MSC) provided by the software.

\section{Skin penetration/permeation study}

This assay was performed according to the study by Serafini et $\mathrm{al}^{31}$ with modifications. The in vitro permeation studies were conducted using porcine ear skin as membrane. The membrane samples were cleaned by removing the hair and adipose tissue. Only skin samples between 1.8 and $2.1 \mathrm{~mm}$ thick, as measured with a dial thickness gauge $\left(\mathrm{N}^{\circ} .7301^{\circledR}\right.$; Mitutoyo, Kawasaki, Japan), were used. The samples were stored at $-4^{\circ} \mathrm{C}$ in aluminum foil until their use. The permeation study was performed with an automatic Franz-type diffusion cell (MicroettePlus Multi-Group ${ }^{\circledR}$; Hanson Research Corporation, Chatsworth, CA, USA) during $24 \mathrm{~h}$. The receptor medium used to guarantee sink condition was $7 \mathrm{~mL}$ of an aqueous solution of ethanol $50 \%(\mathrm{v} / \mathrm{v})$. The formulations (Hst-loaded LNCs and free drug; $0.5 \mathrm{~mL}$ ) were applied to the membranes (diffusion area: $1.77 \mathrm{~cm}^{2}$ ) following the principle of infinite dose and remained in contact with the membrane throughout the experiment time. Samples from the receptor compartments were collected at the end of the experiment time $(24 \mathrm{~h})$. The cells were occluded with a glass cover. The acceptor phase was maintained under constant stirring at a temperature of $32^{\circ} \mathrm{C}$ for the entire experiment. Samples of $1 \mathrm{~mL}$ were withdrawn from the acceptor phase automatically into vials in determined intervals up to $24 \mathrm{~h}$ and injected in the HPLC $(20 \mu \mathrm{L})$. The collected aliquots were replaced with fresh solution. At the end of $24 \mathrm{~h}$, the excess formulations on the surface of the skin were removed with paper. The stratum corneum was obtained by the tape-stripping technique, using 18 tape stripes ( $3 \mathrm{M}^{\circledR}$ Sumaré, São Paulo, Brazil). The epidermis and dermis were separated by heat separation at $60^{\circ} \mathrm{C}$ for 45 seconds followed by removal with a scalpel. Hst was extracted with acetonitrile from all layers of the skin: skin surface $(6 \mathrm{~mL})$, stratum corneum $(4 \mathrm{~mL})$, epidermis $(6 \mathrm{~mL})$ and dermis $(6 \mathrm{~mL})$. The extraction was performed by $2 \mathrm{~min}$ of vortex followed by $30 \mathrm{~min}$ of sonication (USC-2850A model, Unique, 25 kHz, 220 W; Indaiatuba, São Paulo, Brazil). The samples were filtered into vials ( $0.45 \mathrm{~mm}$, Merck Millipore ${ }^{\circledR}$; Darmstadt, Germany) before HPLC analysis. All results are expressed as mean \pm standard deviation $(n=6)$.

\section{Impregnation of the formulations in fibers}

Impregnation of the formulations in the fabrics followed a slightly modified version of the method described by Forgearini et al. ${ }^{27}$ The Hst-loaded LNCs in aqueous dispersion (LNC-Hst) were sprayed onto a $6 \times 6 \mathrm{~cm}^{2}$ piece of fabric three times (cotton or PA). In parallel, a drug hydroalcoholic solution (free-Hst: $0.5 \mathrm{mg} / \mathrm{mL}$ ) was also applied under the same conditions to compare the formulations. For the purpose of estimating the amount of Hst after spraying the product on the fabrics, the relative density of each product, as well as the weight of each fabric sample before and after spraying (LNC-Hst and free-Hst), was determined. The relative density was performed using a $10 \mathrm{~mL}$ pycnometer, comparing to water $(\mathrm{d}=1 \mathrm{~g} / \mathrm{mL})$. After the determination of density, the amount of Hst on fabrics was calculated.

\section{Hst content in fabrics}

The fabrics containing the formulations were cut and placed in tubes containing acetonitrile, which were separately subjected to vortex for $5 \mathrm{~min}$ and then to ultrasound for $30 \mathrm{~min}$ to extract the Hst. After dissolving the materials with acetonitrile, the solution was filtrated and analyzed by HPLC. All analyses were performed in triplicate batches $(n=3)$.

\section{Washing procedure}

This test was performed to analyze the adhesion of Hst to the fabrics. After spraying the formulations onto cotton or PA, 
the fabrics were washed either one or five times. This test was based on the method previously described by Forgearini et $\mathrm{al}^{27}$ in which the samples were introduced into a recipient containing $500 \mathrm{~mL}$ of a $2 \mathrm{~g} / \mathrm{L}$ soap (Savon de Marseille) solution. The recipient was kept in a thermostat at $30^{\circ} \mathrm{C}$. The fabrics were stirred for $10 \mathrm{~min}$ at $200 \mathrm{rpm}$. Subsequently, they were removed and washed two times in distilled water under the same conditions described earlier. After one and five washes, the fabrics were removed and dried at room temperature. For the analysis of the amount of Hst in fabrics after washing, the same methodology described in the "Washing procedure" section was used. The analysis was performed in triplicate $(n=3)$.

\section{Scanning electron microscopy}

To verify the morphology of the fabrics before and after the impregnation with the formulations, as well as to observe the structure of the textile fibers after washing, microscopic analysis of each piece of fabric was performed. The analyzes were carried out using electron microscopy scanning equipment (Scanning Microscope Jeol-JSM-5800) with magnifications of 3,000 times and 5,500 times. These studies were carried out at the Centro de Microscopia Eletrônica of the Universidade Federal do Rio Grande do Sul (Center of Electron Microscopy of the Federal University of Rio Grande do Sul).

\section{Statistical analysis}

Data were evaluated using GraphPad Prism version 5.0 software. The tests used were analysis of variance followed by Tukey's test; differences were considered significant when $P<0.05$.

\section{Results and discussion}

Figure 1 shows the HPLC-DAD Hst chromatographic profile at $290 \mathrm{~nm}$. The Hst peak showed a retention time of $7.5 \mathrm{~min}$. The developed HPLC method was validated by examining linearity, LOD, LOQ, precision, accuracy and robustness. The $r$-value obtained from the data of five concentrations was $>0.999$, verifying the linearity of the equation. The LOD and LOQ were 0.88 and $0.93 \mu \mathrm{g} / \mathrm{mL}$, respectively, indicating that the developed method exhibited high sensitivity. In the intraday variability test, the RSD values were lower than $0.05 \%$. In the interday variability test, the RSDs were 0.38 and $0.49 \%$ at days 1 and 2, respectively. The RSD values of precision assays indicated that the developed method is sufficiently precise. The A\% and RSD of low, medium and high concentrations were $99.99 \% \pm 0.075 \%, 99.97 \% \pm 0.034 \%$ and $100.01 \% \pm 0.071 \%$, respectively, demonstrating that the

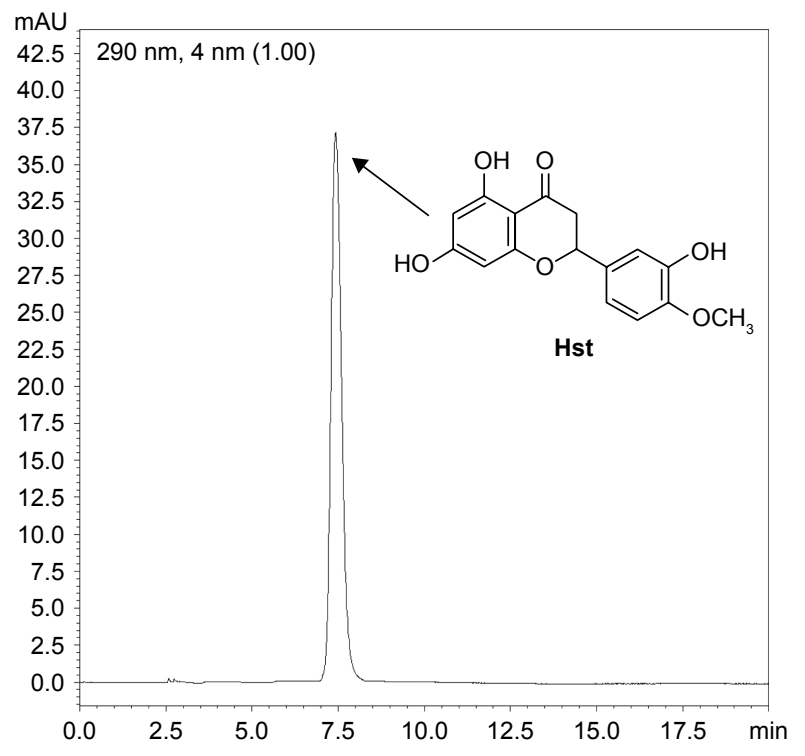

Figure I Hst chromatogram obtained by HPLC-DAD (290 nm). Abbreviations: DAD, diode array detector; HPLC, high-performance liquid chromatography; Hst, hesperetin.

analytical method was accurate. No significant change was observed with the change of analytical column and flow rate, showing that the method is robust under the evaluated conditions.

The nanocapsule formulations showed an opalescent white color and a bluish reflection because of the Tyndall effect as has previously been reported ${ }^{32}$ for lipid-core nanoparticles of quetiapine and ${ }^{21}$ for LNC formulations similar to those in our study. Furthermore, the mean particle size was $204 \pm 1 \mathrm{~nm}$ with a low PDI (0.14 \pm 0.02$)$. Zeta potential was $-11.8 \pm 0.9 \mathrm{mV}$. The electrostatic repulsion resulting from surface charge density $^{33}$ is in general the mechanism for a kinetic stabilization of the hydrophobic colloidal phase dispersed in water. In this case, the negative zeta potential value is close to zero as a consequence of the chemical composition (polyester coated by polysorbate 80 ), in which stabilization mechanism is based on the steric hindrance effect. ${ }^{13,34}$ The reproducibility of size distribution batch-to-batch shows that the formulation is kinetically stable, ie, the system has a delayed aggregation phase separation. The PCL used in the nanocapsule formulation has $-\mathrm{COOH}$ end groups, which can present an acidbase balance forming carboxylates, rendering the surface negatively charged. Raber et $\mathrm{al}^{35}$ have shown that negatively charged nanoparticles interact more effectively with the skin surface, facilitating follicular uptake. The particle diameter profile determined by laser diffraction showed a volumeweighted mean diameter ( $D$ [4.3]) of $233 \pm 8 \mathrm{~nm}, \mathrm{~d}_{\mathrm{v}}(0.5)$ of $198 \pm 8 \mathrm{~nm}$ and polydispersity (span value) of $1.75 \pm 0.02$. The results demonstrated that the developed nanocapsules 
have adequate mean diameters and narrow particle size distributions in the current study. Similar physicochemical properties with respect to size distribution and zeta potential have been reported in other studies for the same type of polymeric nanocarrier. Oliveira et $\mathrm{al}^{36}$ in a study on methotrexate diethyl ester (MTX $\left.(\mathrm{OEt})_{2}-\mathrm{LNC}^{0.5}\right)$ formulation at a similar LNC volume fraction had median diameters, based on a volume of $\mathrm{d}_{\mathrm{v}}(0.5)=160 \mathrm{~nm}$, and Friedrich et $\mathrm{al}^{37}$ studying tacrolimus-loaded LNCs had a volume of $d_{v}(0.5)=192 \mathrm{~nm}$ and a zeta potential of $-13.05 \mathrm{mV}$. PCL at the particle-water

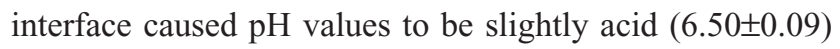
due to the presence of terminal carboxylic acid groups in the polymer backbone. ${ }^{19,38}$

The drug content determined for the Hst-loaded LNCs was $0.46 \pm 0.01 \mathrm{mg} / \mathrm{mL}$. Moreover, the results of the EE showed that almost $98.81 \% \pm 0.28 \%$ of the Hst molecules were entrapped in the nanocapsules, which is expected for hydrophobic molecules such as Hst. These data suggest a strong affinity between the flavonoid and LNCs. In general, the drug lipophilicity is the main parameter in terms of drug partitioning among the pseudo-phases of the colloidal dispersions. ${ }^{39}$ According to the algorithm proposed by Oliveira et $\mathrm{al}^{36}$ drugs presenting positive $\log \mathrm{D}$ values, such as that of Hst (2.82), have an LNC drug distribution mechanism type III, indicating its higher affinity for the lipophilic phase. TEM showed a core-corona structure of spherical nanoparticles and confirmed their nanometric size (Figure 2). Similar findings have been described by Fathi et $\mathrm{al}^{23}$ and

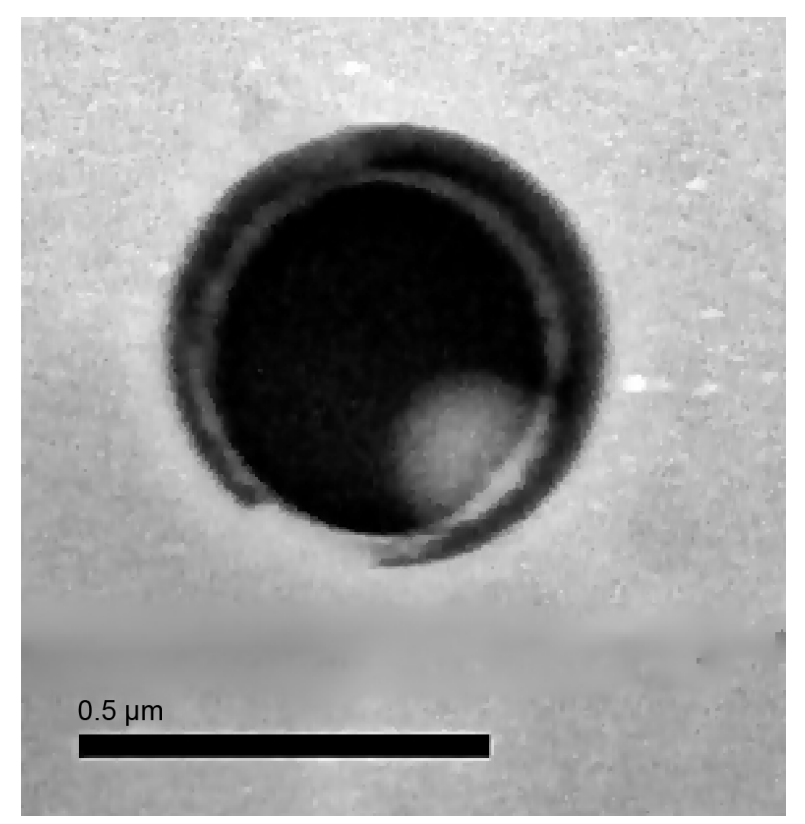

Figure 2 Transmission electron micrograph of Hst-loaded LNC. Abbreviations: Hst, hesperetin; LNC, lipid-core nanocapsule.
Panek et $\mathrm{al}^{40}$ for other Hst-loaded nanocarrier formulations composed of polysorbate $80 /$ glycerol monostearate/compritol and polystyrene/tetrahydrofuran/water, poly(methyl methacrylate)/acetone/water, respectively.

The in vitro release experiment for free-Hst and LNCHst was carried out under sink condition by dialysis method with ethanol $10 \%(\mathrm{v} / \mathrm{v})$ as the release medium. The results of the study are shown in Figure 3. The free-Hst showed a fast release $(104.96 \% \pm 12.83 \%)$ after $4 \mathrm{~h}$. In contrast, after $24 \mathrm{~h}$, only $69.90 \% \pm 1.33 \%$ of the Hst was released from the LNCHst formulation, indicating a sustained release. Moreover, the initial burst corroborates the proposition of encapsulation mechanism type III, in which the drug is partially located in the polymer wall of the LNC.

According to the highest correlation coefficient $(r)$ and MSC, the best adjustment proved to be the zeroorder model $(\mathrm{k} 1: 0.0696 \pm 0.0054 \mathrm{~h}$ and $0.7951 \pm 0.0266 \mathrm{~h}$; $r: 0.9905 \pm 0.0081$ and $0.9821 \pm 0.0013$; MSC: $0.5572 \pm 0.1880$ and $1.4187 \pm 0.4005$ ) for the LNC-Hst and free-Hst, respectively. Based on the results described earlier, it was possible to observe the release profile of the drug at a constant rate (regardless of the concentration). According to a review by Siepmann and Siepmann, ${ }^{41}$ perfect sink conditions are maintained throughout the release period and drug release is primarily controlled by diffusion through the carrier matrix. The half-life time of drug release from the LNC-Hst was $9.9483 \mathrm{~h}$, confirming the high efficiency of the encapsulation already discussed in this study, while the free drug showed a significantly faster diffusion $\left(t_{1 / 2}=0.8716 \mathrm{~h}\right.$ ). Regarding the type of release, the LNC-Hst obeyed Fickian diffusion and comprised a reservoir system with a drug depot surrounded by a release-controlling barrier membrane formed by PCL. ${ }^{38,41}$

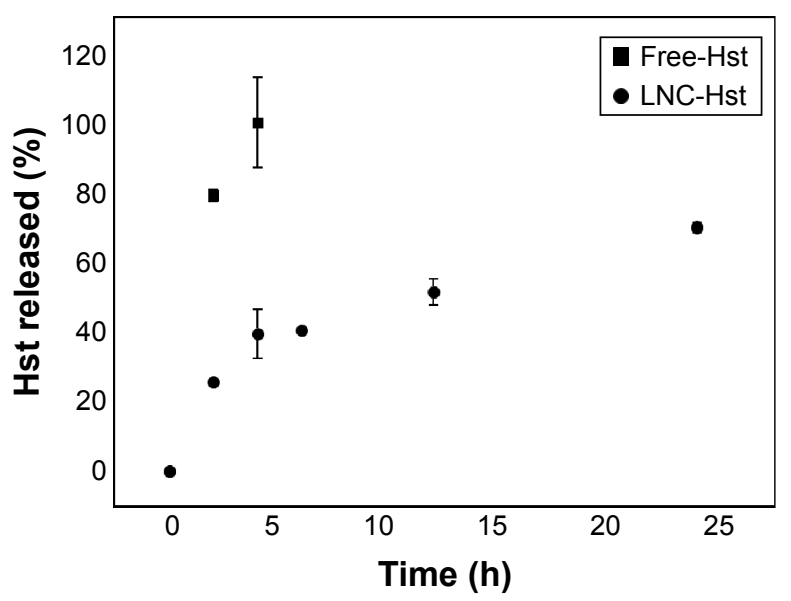

Figure 3 In vitro release profiles of Hst from the dialysis of Hst-loaded LNCs (LNC-Hst, circle) and ethanolic solution of Hst (free-Hst, square; $n=3$ ). Abbreviations: Hst, hesperetin; LNC, lipid-core nanocapsule. 
Nanocarriers presenting sustained release of the drug can act as good drug delivery systems to the skin, targeting the drug and improving drug penetration. ${ }^{42}$ Cutaneous penetration of free and nanoencapsulated Hst was determined from successive tape stripping of the stratum corneum and extraction of the drug from the skin layers. Our results (Figure 4) showed significantly higher quantities $(P<0.05)$ of Hst from LNC-Hst $\left(6.31 \pm 0.10 \mu \mathrm{g} / \mathrm{cm}^{2}\right)$ than from free-Hst $\left(5.52 \pm 0.21 \mu \mathrm{g} / \mathrm{cm}^{2}\right)$ in the stratum corneum. In contrast, we observed significantly $(P<0.05)$ higheramounts of Hst fromLNC$\operatorname{Hst}\left(5.94 \pm 0.07 \mu \mathrm{g} / \mathrm{cm}^{2}\right)$ than from the free-Hst $\left(7.23 \pm 0.45 \mu \mathrm{g} / \mathrm{cm}^{2}\right)$ in the epidermis. In the dermis, the amounts of Hst from LNCHst $\left(7.63 \pm 0.32 \mu \mathrm{g} / \mathrm{cm}^{2}\right)$ and from free-Hst $\left(8.23 \pm 0.21 \mu \mathrm{g} / \mathrm{cm}^{2}\right)$ were statistically similar $(P>0.05)$. The results can be explained by the reservoir characteristic of the LNC-Hst, which can control the drug penetration, leading to a prolonged amount of Hst being available to the dermis, as previously reported for Eudragit ${ }^{\circledR}$ polymeric nanocapsules. ${ }^{18}$ The likely permeated amount of the drug is considered the receptor fluid, and we observed a significantly $(P<0.05)$ higher amount of Hst from free-Hst $\left(3.12 \pm 0.14 \mu \mathrm{g} / \mathrm{cm}^{2}\right)$ compared to the drug permeated from LNC-Hst $\left(1.95 \pm 0.08 \mu \mathrm{g} / \mathrm{cm}^{2}\right)$. These results corroborate the drug release assay, which demonstrated that the free drug diffuses rapidly from its solution, while it is released in a controlled manner from LNC-Hst. In recent years, studies have reported that the small size of nanocapsules enables them to penetrate cells and deliver drugs intracellularly without suffering extracellular degradation. ${ }^{43,44}$ Moreover, controlled release systems can prolong the release and increase the contact time between the drug and the skin. ${ }^{21}$ In addition, LNC-Hst increased the retention of the drug in the stratum corneum, possibly due to its rigid structure, ${ }^{45}$ which maintains controlled release of the drug in the skin. LNCs lose their shape a few hours after cutaneous application due to the interaction between the lipids in the nanoparticles and the stratum corneum. ${ }^{21,46}$

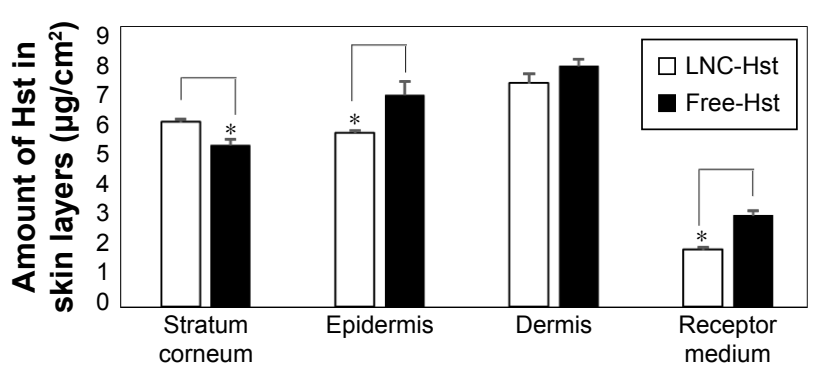

Figure 4 Skin penetration and permeation profile of nanoencapsulated Hst (LNC-Hst) and free drug (free-Hst).

Note: *Represents statistical significance $(P<0.05)$.

Abbreviations: Hst, hesperetin; LNC, lipid-core nanocapsule.
As this formulation shows promise for use in a topical application, it was impregnated into textile fibers as an alternative to conventional formulations for the treatment of CVI. There are some studies that have used different fabrics (PA, polyurethane and viscose) impregnated with drugs (troxerutin and aescin) for the treatment of CVI. However, these studies used cyclodextrins as the drug delivery platform, ${ }^{25,26,29}$ while our study used LNCs to deliver Hst for the treatment of this disease. We impregnated our formulation into either natural cotton or synthetic fiber (PA, made from $\varepsilon$-caprolactam or hexamethylenediamine and adipic acid) used in fabrication of elastic compression stockings. ${ }^{47,48}$ The relative density of the formulations needed to be determined to calculate the necessary Hst content to impregnate in the fibers. The fibers were weighed before and after the formulations were applied (three times). The values of the densities of the LNC-Hst and freeHst were 1.03 and $0.93 \mathrm{mg} / \mathrm{mL}$, respectively. With respect to the Hst content of the fabrics, we could observe that the cotton fiber contained less free-Hst $\left(93,033.91 \pm 2,684.81 \mu \mathrm{g} / \mathrm{cm}^{2}\right)$ and LNC-Hst $\left(387,633.40 \pm 8,331.20 \mu \mathrm{g} / \mathrm{cm}^{2}\right)$ when compared with the PA fiber (free-Hst: $358,577.2 \pm 1,686.82 \mu \mathrm{g} / \mathrm{cm}^{2}$ and LNC-Hst: 1,281,281 $\pm 5,074.31 \mu \mathrm{g} / \mathrm{cm}^{2}$; Figure 5). The better impregnation profile of the PA fiber samples, especially in relation to LNC-Hst, can be explained because polymers have the characteristic of binding together through weaker intermolecular forces such as van der Waals forces, hydrogen bonding or dipole-dipole interaction. Moreover, the polymer-polymer interaction between the fabric and LNC-Hst polymers is quite effective. ${ }^{48}$ This means that one interaction between the two polymers is sufficient to produce a high level of adhesiveness in the nanoparticles. This type of interaction is a hydrogen bond between the $\mathrm{H}$ donor $\mathrm{PA}$ from the $\mathrm{NH}$ group and the $\mathrm{C}=\mathrm{O}$ present in the $\mathrm{PCL}$ receptor.

After washing the fabrics, it was observed that the PA with the free-Hst and the LNC-Hst lost less drug over the

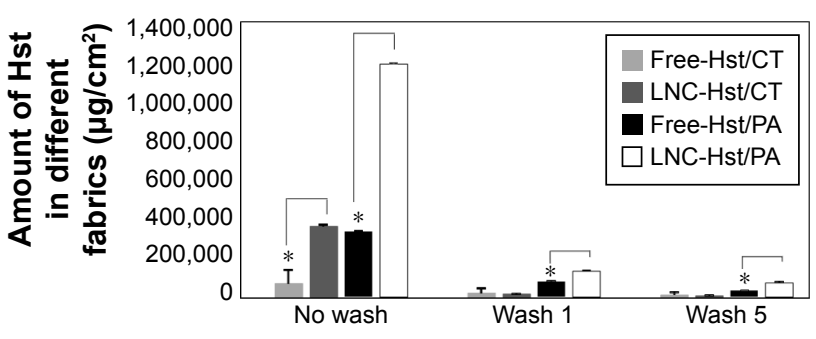

Figure 5 Quantification of nanoencapsulated Hst (LNC-Hst) and hydroalcoholic solution (free-Hst) impregnated in CT and PA fibers before after one and five washes.

Note: $* P<0.05$ free-Hst versus LNC-Hst.

Abbreviations: CT, cotton; Hst, hesperetin; LNC, lipid-core nanocapsule; PA, polyamide. 


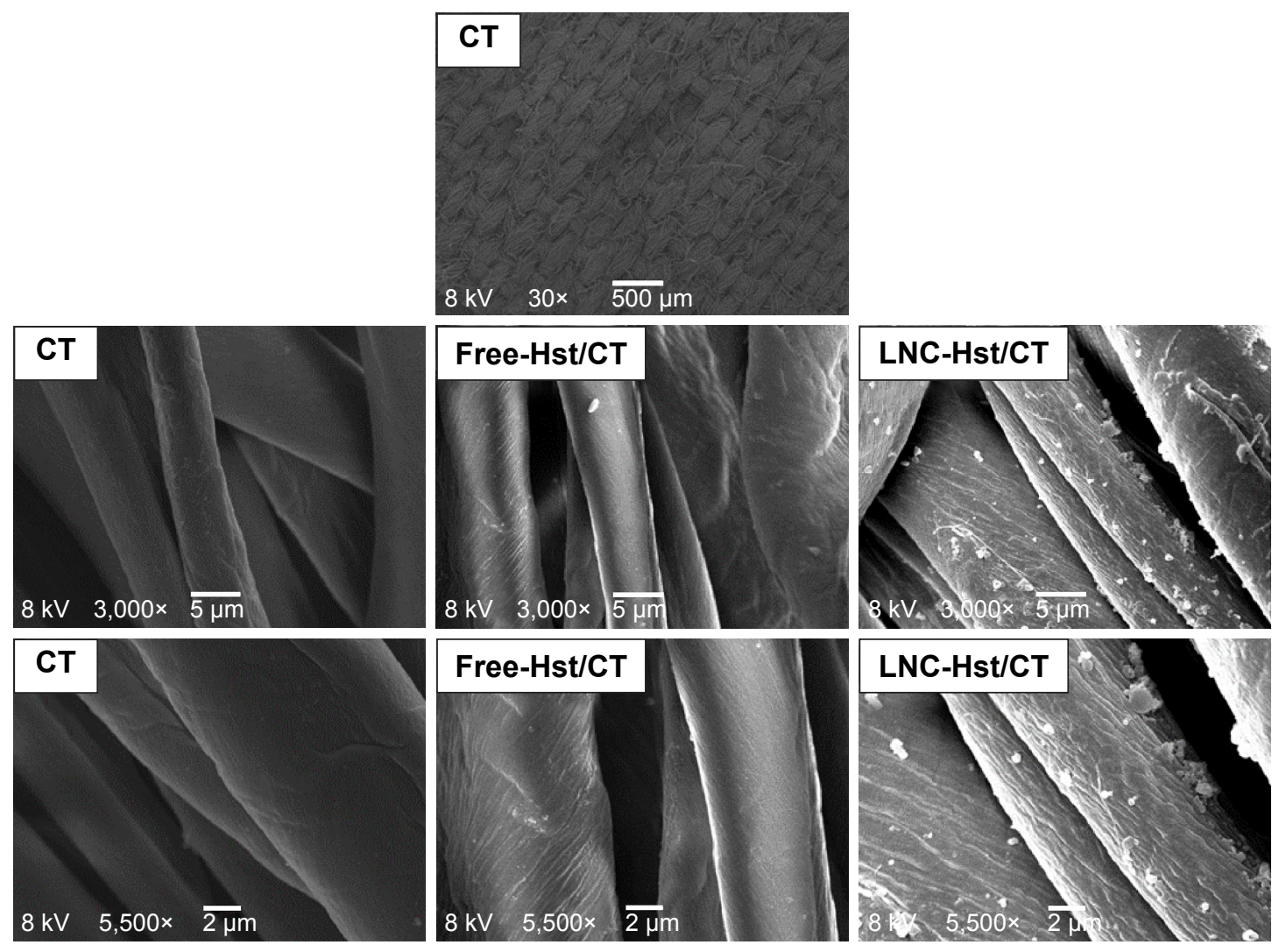

Figure 6 Scanning electron micrographs of nanoencapsulated Hst (LNC-Hst) and hydroalcoholic solution (free-Hst) impregnated in CT fiber at different magnifications. Abbreviations: CT, cotton; Hst, hesperetin; LNC, lipid-core nanocapsule.

five washes and was therefore chosen as the fabric to be used in the rest of the study. After one wash, the PA had 2.54 times more LNC-Hst than the cotton. After five washes, the PA had 2.80 times more LNC-Hst than the cotton. Scanning electron microscopy images (Figures 6-9) corroborate these data, showing a better impregnation profile in samples of the PA fibers. Furthermore, at $30 \times$ magnification, it is possible to observe the structure of the fibers. Cotton fibers are more closed, while PA is more open with space for better impregnation. Moreover, bigger particles were observed

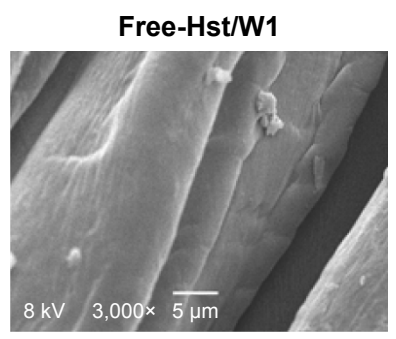

LNC-Hst/W1

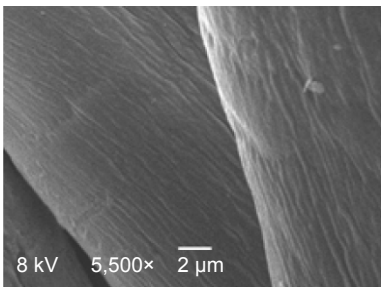

Free-Hst/W5

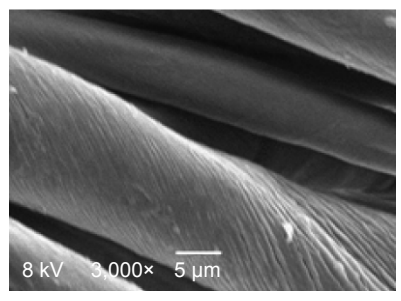

LNC-Hst/W5

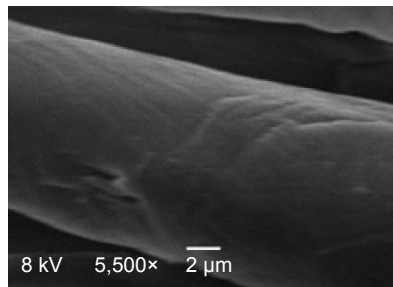

LNC-Hst/W1

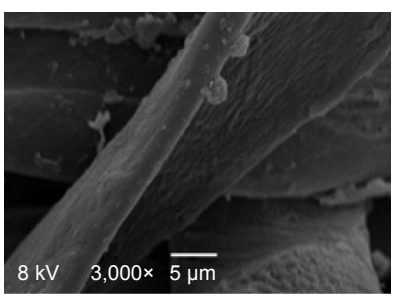

LNC-Hst/W1

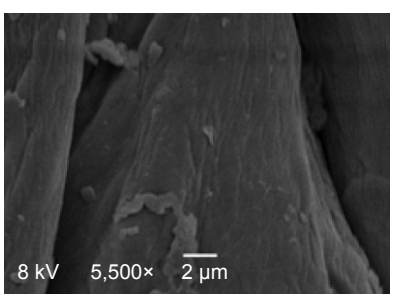

LNC-Hst/W5

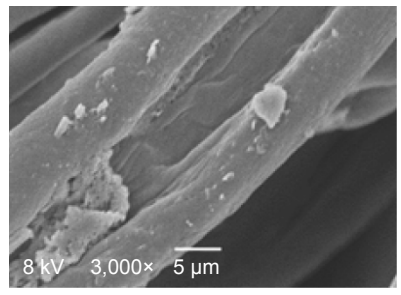

LNC-Hst/W5

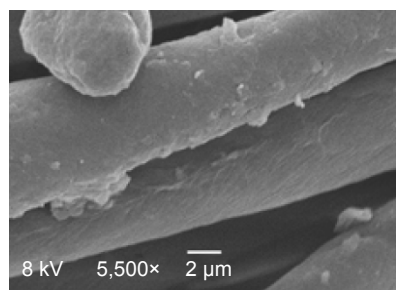

Figure 7 Scanning electron micrographs of nanoencapsulated Hst (LNC-Hst) and hydroalcoholic solution (free-Hst) impregnated in CT fiber after one (WI) and five washes (W5).

Abbreviations: CT, cotton; Hst, hesperetin; LNC, lipid-core nanocapsule. 

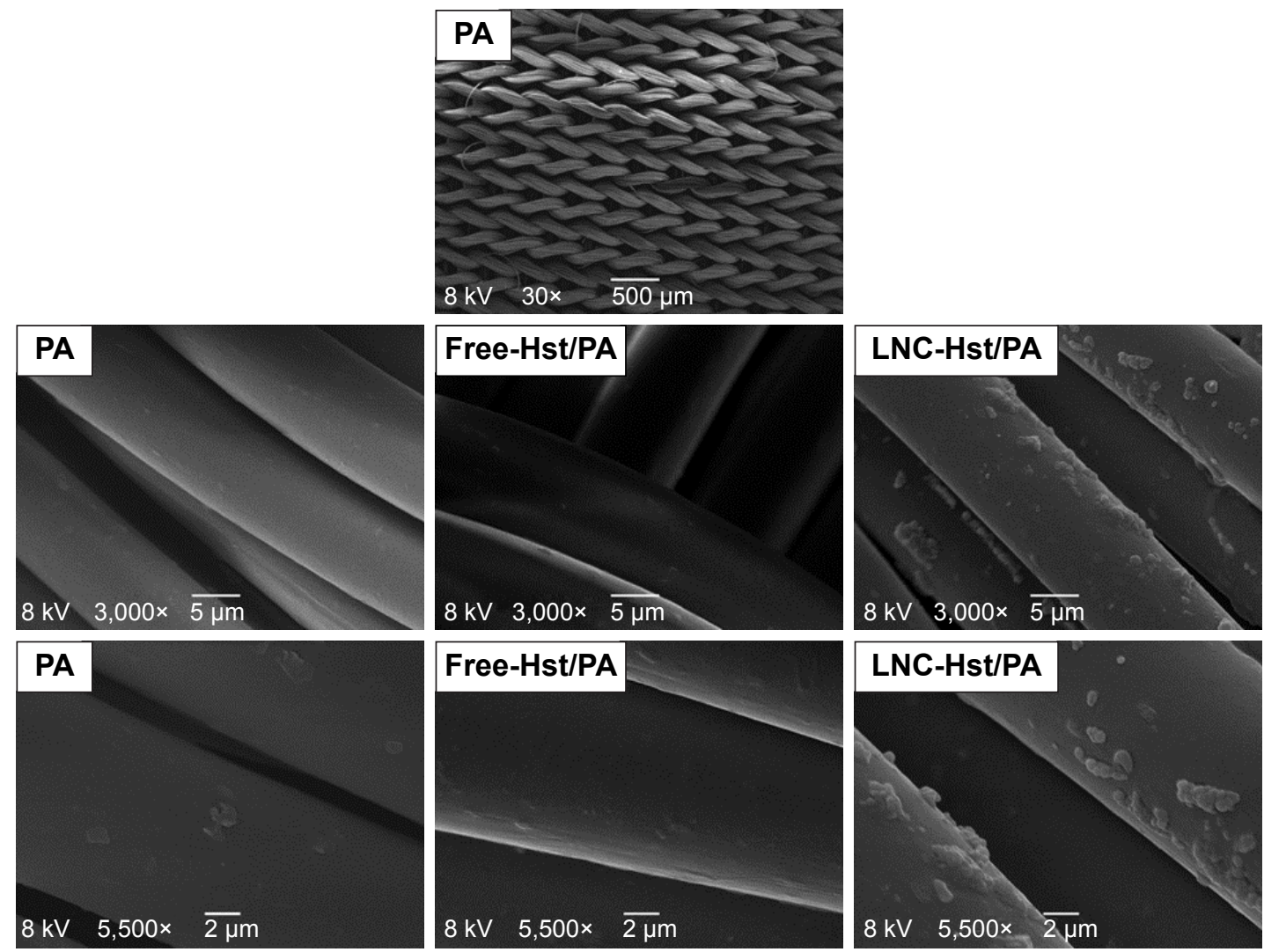

Figure 8 Scanning electron micrographs of nanoencapsulated Hst (LNC-Hst) and hydroalcoholic solution (free-Hst) impregnated in PA fiber at different magnifications. Abbreviations: Hst, hesperetin; LNC, lipid-core nanocapsule; PA, polyamide.

in the water in which the cotton had been washed than the characterization (D [4.3] and d [0.5]). It is noteworthy that particles with micrometric size were observed in the cotton (data observed by dynamic light scattering; Table 1), suggesting that such particles adhere to the cotton fibers in the form of agglomerates (as shown in the scanning electron micrographic images; Figure 7), since there is not enough room for individualized adherence of particles, as is the case of PA (Figure 9 and Table 1). The high negative zeta potential value of LNC-Hst/PA can be attributed to PA alone

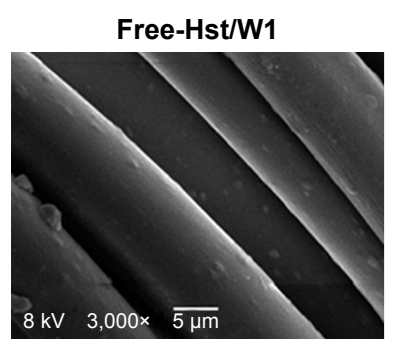

Free-Hst/W1

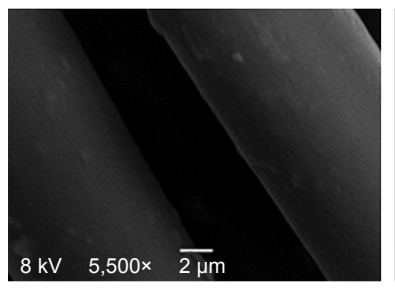

Free-Hst/W5

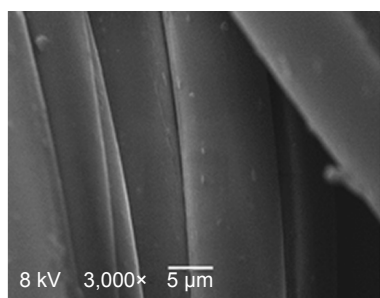

Free-Hst/W5

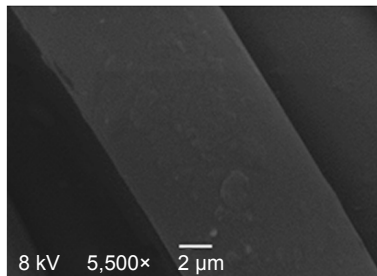

LNC-Hst/W1

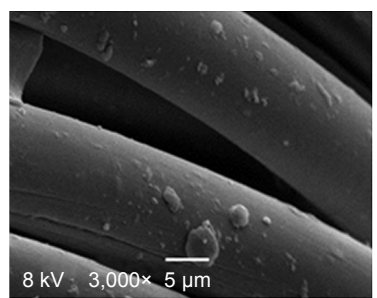

LNC-Hst/W1

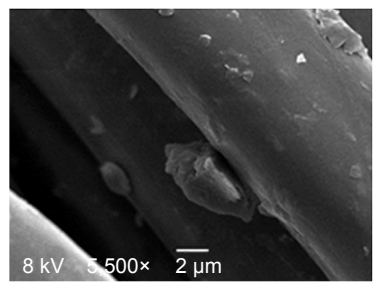

LNC-Hst/W5

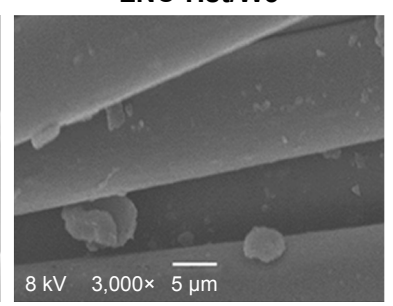

LNC-Hst/W5

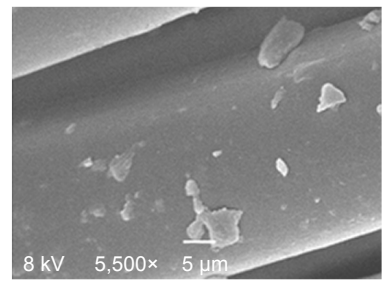

Figure 9 Scanning electron micrographs of nanoencapsulated Hst (LNC-Hst) and hydroalcoholic solution (free-Hst) impregnated in PA fiber after one (WI) and five washes (W5).

Abbreviations: Hst, hesperetin; LNC, lipid-core nanocapsule; PA, polyamide. 
Table I Size distribution obtained by dynamic light scattering and zeta potential of LNC-Hst impregnated in different fabrics after water washes

\begin{tabular}{|c|c|c|c|c|c|c|}
\hline Sample & $\begin{array}{l}\text { Size } \\
(\mathrm{nm})\end{array}$ & $\begin{array}{l}D(4.3) \\
(\mathrm{nm})\end{array}$ & $\begin{array}{l}d(v, 0.5) \\
(n m)\end{array}$ & Span & $\begin{array}{l}\text { Zeta } \\
\text { potential } \\
(\mathrm{mV})\end{array}$ & PDI \\
\hline LNC-Hst/CT & I,I58.00 & 473.00 & 360.00 & 2.36 & -3.66 & 0.932 \\
\hline LNC-Hst/PA & 863.40 & 160.00 & 145.00 & 1.18 & -28.3 & 0.824 \\
\hline
\end{tabular}

Notes: D (4.3), diameter of particles; D ( $v, 0.5)$, diameter of $50 \%$ of particles based on volume; Span, width of the size distribution.

Abbreviations: $\mathrm{CT}$, cotton; Hst, hesperetin; LNC, lipid-core nanocapsule; PA, polyamide; PDI, polydispersity index.

as it has a high negative zeta potential as has been shown by Espinosa-Jiménez et al. ${ }^{49}$

The permeation/penetration profile (Figure 10) of the PA impregnated with LNC-Hst using porcine ear skin showed that drug impregnated in the nanocapsules permeated PA, once they have been quantified in the dermis without significant difference in this layer as well as on the receptor medium ( $50 \%$ ethanol). The nanocapsules and free Hst were able to leave the fabric and permeate the skin layers with a significant difference only in the stratum corneum and epidermis. After $24 \mathrm{~h}$ there was an almost identical amount of LNC-Hst and free-Hst in the PA fibers $\left(0.25 \pm 0.01\right.$ and $0.24 \pm 0.02 \mu \mathrm{g} / \mathrm{cm}^{2}$, respectively), indicating that LNC-Hst was able to permeate/penetrate the fabric to skin layers because is a controlled release system that hand had a significant difference to the drug release free immediate. Thus, PA is a good platform for the release of nanoencapsulated Hst in the treatment of diseases of the circulatory system, as discussed in the current study. Radu et a ${ }^{29}$ highlighted that the main factors that contribute to the permeation of drugs to the skin are sweating, friction and skin enzymes.

\section{Conclusion}

The LNCs were successfully formulated showing suitable size and distribution characteristics. Furthermore, these nanocapsules presented controlled release through a Fickian

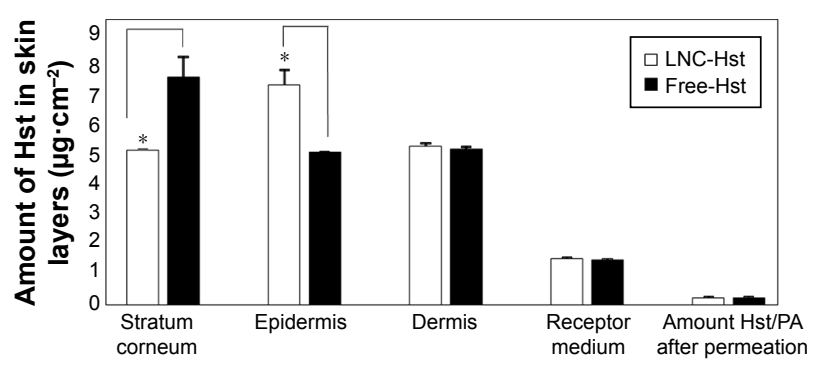

Figure 10 Skin penetration/permeation profile of nanoencapsulated Hst (LNC-Hst) and free drug (free-Hst) impregnated in PA.

Note: *Represents statistical significance $(P<0.05)$.

Abbreviations: Hst, hesperetin; LNC, lipid-core nanocapsule; PA, polyamide. diffusion release model. Adhesion of the nanocapsules to the fabrics after washing was found to be best in the PA. PA fabric impregnated with Hst nanocapsules was able to carry the drug into the skin. These data support future clinical trials of PA/LNC-Hst fabric for the treatment of CVI.

\section{Acknowledgment}

The authors thank the CAPES, FAPITEC/SE and CNPq (Brazil) for their financial support.

\section{Disclosure}

The authors report no conflicts of interest in this work.

\section{References}

1. Roohbakhsh A, Parhiz H, Soltani F, Rezaee R, Iranshahi M. Molecular mechanisms behind the biological effects of hesperidin and hesperetin for the prevention of cancer and cardiovascular diseases. Life Sci. 2015;124:64-74.

2. Parhiz H, Roohbakhsh A, Soltani F, Rezaee R, Iranshahi M. Antioxidant and anti-inflammatory properties of the citrus flavonoids hesperidin and hesperetin: an updated review of their molecular mechanisms and experimental models. Phytother Res. 2015;29(3):323-331.

3. Adelli GR, Hingorani T, Punyamurthula N, Balguri SP, Majumdar S. Evaluation of topical hesperetin matrix film for back-of-the-eye delivery. Eur J Pharm Biopharm. 2015;92:74-82.

4. Gimenez-Bastida JA, Gonzalez-Sarrias A, Vallejo F, Espin JC, TomasBarberan FA. Hesperetin and its sulfate and glucuronide metabolites inhibit TNF-[small alpha] induced human aortic endothelial cell migration and decrease plasminogen activator inhibitor-1 (PAI-1) levels. Food Funct. 2016;7(1):118-126.

5. Liu Y, Niu L, Cui L, et al. Hesperetin inhibits rat coronary constriction by inhibiting $\mathrm{Ca} 2+$ influx and enhancing voltage-gated $\mathrm{K}+$ channel currents of the myocytes. Eur J Pharmacol. 2014;735:193-201.

6. Jin Y-R, Han X-H, Zhang Y-H, et al. Antiplatelet activity of hesperetin, a bioflavonoid, is mainly mediated by inhibition of PLC- $\gamma 2$ phosphorylation and cyclooxygenase-1 activity. Atherosclerosis. 2007; 194(1):144-152.

7. Arya A, Khandelwal K, Ahmad H, et al. Co-delivery of hesperetin enhanced bicalutamide induced apoptosis by exploiting mitochondrial membrane potential via polymeric nanoparticles in a PC-3 cell line. RSC Adv. 2016;6(7):5925-5935.

8. Menezes PP, Serafini MR, Santana BV, et al. Solid-state $\beta$-cyclodextrin complexes containing geraniol. Thermochim Acta. 2012;548:45-50.

9. Menezes PP, Serafini MR, Quintans-Júnior LJ, et al. Inclusion complex of (-)-linalool and b-cyclodextrin. $J$ Therm Anal Calorim. 2014;115: 2429-2437.

10. Menezes Pdos P, Araujo AA, Doria GA, et al. Physicochemical characterization and analgesic effect of inclusion complexes of essential oil from Hyptis pectinata L. Poit leaves with beta-cyclodextrin. Curr Pharm Biotechnol. 2015;16(5):440-450.

11. Yang L-J, Xia S, Ma S-X, et al. Host-guest system of hesperetin and $\beta$-cyclodextrin or its derivatives: preparation, characterization, inclusion mode, solubilization and stability. Mater Sci Eng C. 2016;59: 1016-1024.

12. Frank LA, Contri RV, Beck RCR, Pohlmann AR, Guterres SS. Improving drug biological effects by encapsulation into polymeric nanocapsules. Wiley Interdiscip Rev Nanomed Nanobiotechnol. 2015;7(5):623-639.

13. Mathes C, Melero A, Conrad P, et al. Nanocarriers for optimizing the balance between interfollicular permeation and follicular uptake of topically applied clobetasol to minimize adverse effects. J Control Release. 2016;223:207-214.

14. Bulcao RP, de Freitas FA, Dallegrave E, et al. In vivo toxicological evaluation of polymeric nanocapsules after intradermal administration. Eur J Pharm Biopharm. 2014;86(2):167-177. 
15. Al Shaal L, Mishra PR, Muller RH, Keck CM. Nanosuspensions of hesperetin: preparation and characterization. Pharmazie. 2014; 69(3):173-182.

16. Bitencourt PER, Ferreira LM, Cargnelutti LO, et al. A new biodegradable polymeric nanoparticle formulation containing Syzygium cumini: phytochemical profile, antioxidant and antifungal activity and in vivo toxicity. Ind Crops Prod. 2016;83:400-407.

17. WHO. Adherence to Long-Term Therapies: Evidences for Action. Geneva: WHO; 2003.

18. Contri RV, Katzer T, Ourique AF, et al. Combined effect of polymeric nanocapsules and chitosan hydrogel on the increase of capsaicinoids adhesion to the skin surface. J Biomed Nanotechnol. 2014;10(5): 820-830.

19. Schaffazick SR, Guterres SS, Freitas Ld L, Pohlmann AR. Caracterização e estabilidade físico-química de sistemas poliméricos nanoparticulados para administração de fármacos [Physicochemical characterization and stability of the polymeric nanoparticle systems for drug administration] Química Nova. 2003;26:726-737. Portuguese.

20. Mora-Huertas CE, Fessi H, Elaissari A. Polymer-based nanocapsules for drug delivery. Int J Pharm. 2010;385(1-2):113-142.

21. Venturini CG, Bruinsmann FA, Contri RV, et al. Co-encapsulation of imiquimod and copaiba oil in novel nanostructured systems: promising formulations against skin carcinoma. Eur J Pharm Sci. 2015;79:36-43.

22. Guterres SS, Alves MP, Pohlmann AR. Polymeric nanoparticles, nanospheres and nanocapsules, for cutaneous applications. Drug Target Insights. 2007;2:147-157.

23. Fathi M, Varshosaz J. Novel hesperetin loaded nanocarriers for food fortification: production and characterization. $J$ Funct Foods 2013;5(3):1382-1391.

24. Shete G, Pawar YB, Thanki K, Jain S, Bansal AK. Oral bioavailability and pharmacodynamic activity of hesperetin nanocrystals generated using a novel bottom-up technology. Mol Pharm. 2015;12(4):1158-1170.

25. Cravotto G, Beltramo L, Sapino S, Binello A, Carlotti ME. A new cyclodextrin-grafted viscose loaded with aescin formulations for a cosmeto-textile approach to chronic venous insufficiency. J Mater Sci Mater Med. 2011;22(10):2387-2395.

26. Nichifor M, Constantin M, Mocanu G, et al. New multifunctional textile biomaterials for the treatment of leg venous insufficiency. J Mater Sci Mater Med. 2009;20(4):975-982.

27. Forgearini JC, Michalowski CB, Assumpção E, Pohlmann AR, Guterres SS. Development of an insect repellent spray for textile based on permethrin-loaded lipid-core nanocapsules. J Nanosci Nanotechnol. 2016;16(2):1301-1309.

28. Yuan G, Cranston R. Recent advances in antimicrobial treatments of textiles. Textile Res J. 2008;78(1):60-72.

29. Radu C-D, Parteni O, Ochiuz L. Applications of cyclodextrins in medical textiles - review. J Control Release. 2016;224:146-157.

30. Jornada DS, Fiel LA, Bueno K, et al. Lipid-core nanocapsules: mechanism of self-assembly, control of size and loading capacity. Soft Matter. 2012;8(24):6646-6655.

31. Serafini MR, Detoni CB, Guterres SS, da Silva GF, de Souza Araujo AA. Determination of in vitro usnic acid delivery into porcine skin using a HPLC method. J Chromatogr Sci. 2015;53(5):757-760.

32. Carreño F, Paese K, Silva CM, Guterres SS, Dalla Costa T. Pre-clinical investigation of the modulation of quetiapine plasma pharmacokinetics and tissues biodistribution by lipid-core nanocapsules. J Pharm Biomed Anal. 2016;119:152-158.
33. Pereira RL, Leites FI, Paese K, et al. Hydrogel containing adapaleneand dapsone-loaded lipid-core nanocapsules for cutaneous application: development, characterization, in vitro irritation and permeation studies. Drug Dev Ind Pharm. 2016;42(12):2001-2008.

34. Benvegnu DM, Barcelos RC, Boufleur N, et al. Haloperidol-loaded polysorbate-coated polymeric nanocapsules decrease its adverse motor side effects and oxidative stress markers in rats. Neurochem Int. 2012;61(5):623-631.

35. Raber AS, Mittal A, Schafer J, et al. Quantification of nanoparticle uptake into hair follicles in pig ear and human forearm. $J$ Control Release. 2014;179:25-32.

36. Oliveira CP, Venturini CG, Donida B, Poletto FS, Guterres SS, Pohlmann AR. An algorithm to determine the mechanism of drug distribution in lipid-core nanocapsule formulations. Soft Matter. 2013;9(4): 1141-1150.

37. Friedrich RB, Coradini K, Fonseca FN, Guterres SS, Beck RC, Pohlmann AR. Lipid-core nanocapsules improved antiedematogenic activity of tacrolimus in adjuvant-induced arthritis model. $J$ Nanosci Nanotechnol. 2016;16(2):1265-1274.

38. Ribeiro RF, Motta MH, Härter APG, et al. Spray-dried powders improve the controlled release of antifungal tioconazole-loaded polymeric nanocapsules compared to with lyophilized products. Mater Sci Eng C. 2016;59:875-884.

39. Stella B, Arpicco S, Rocco F, et al. Encapsulation of gemcitabine lipophilic derivatives into polycyanoacrylate nanospheres and nanocapsules. Int J Pharm. 2007;344(1-2):71-77.

40. Panek J, Filippov SK, Konak C, et al. Polymeric nanoparticles stabilized by surfactants investigated by light scattering, small-angle neutron scattering, and cryo-TEM methods. J Dispersion Sci Technol. 2011;32(6):888-897.

41. Siepmann J, Siepmann F. Mathematical modeling of drug delivery. Int J Pharm. 2008;364(2):328-343.

42. Batheja P, Sheihet L, Kohn J, Singer AJ, Michniak-Kohn B. Topical drug delivery by a polymeric nanosphere gel: formulation optimization and in vitro and in vivo skin distribution studies. $J$ Control Release. 2011;149(2):159-167.

43. Mallipeddi R, Rohan LC. Nanoparticle-based vaginal drug delivery systems for HIV prevention. Expert Opin Drug Deliv. 2010;7(1):37-48.

44. Frank LA, Sandri G, D'Autilia F, et al. Chitosan gel containing polymeric nanocapsules: a new formulation for vaginal drug delivery. Int J Nanomedicine. 2014;9:3151-3161.

45. Fiel LA, Rebelo LM, de Melo Santiago T, et al. Diverse deformation properties of polymeric nanocapsules and lipid-core nanocapsules. Soft Matter. 2011;7(16):7240-7247.

46. Küchler S, Abdel-Mottaleb M, Lamprecht A, Radowski MR, Haag R, Schäfer-Korting $M$. Influence of nanocarrier type and size on skin delivery of hydrophilic agents. Int J Pharm. 2009;377(1-2):169-172.

47. Qin Y. 2 - An overview of medical textile products. Medical Textile Materials. Jiaxing: Woodhead Publishing; 2016:13-22.

48. Qin Y.3 - A brief description of textile fibers. Medical Textile Materials. Jiaxing: Woodhead Publishing; 2016:23-42.

49. Espinosa-Jiménez M, Ortega AO, Weigand RP. Análisis electrocinético y termodinámico del proceso de adsorción de azul ácido 45 sobre tejido de poliamida. Boletín INTEXTER (U.P.C.). 2001;120:37-41.
International Journal of Nanomedicine

\section{Publish your work in this journal}

The International Journal of Nanomedicine is an international, peerreviewed journal focusing on the application of nanotechnology in diagnostics, therapeutics, and drug delivery systems throughout the biomedical field. This journal is indexed on PubMed Central, MedLine, CAS, SciSearch ${ }^{\circledR}$, Current Contents ${ }^{\circledR} /$ Clinical Medicine,

\section{Dovepress}

Journal Citation Reports/Science Edition, EMBase, Scopus and the Elsevier Bibliographic databases. The manuscript management system is completely online and includes a very quick and fair peer-review system, which is all easy to use. Visit http://www.dovepress.com/ testimonials.php to read real quotes from published authors. 